\title{
Introducing Functional Tones for Analysing Action Perception in Manufacturing: Explaining What Affordances Cannot
}

\author{
Erik LAGERSTEDT ${ }^{\mathrm{a}, 1}$ and Ari KOLBEINSSON ${ }^{\mathrm{b}}$ \\ ${ }^{a}$ School of Informatics, University of Skövde, Högskolevägen, Skövde 541 28, Sweden \\ ${ }^{\mathrm{b}}$ School of Engineering, University of Skövde, Högskolevägen, Skövde 541 28, Sweden
}

\begin{abstract}
Functional tones is a concept that originates in theoretical biology and resembles how the concept 'affordances' is used. Both functional tones and affordances are concepts dealing with particularly salient features in an individual's immediate environment. The concept of affordances has proven useful for practitioners of usability and design as it supports intuitive ways of classifying how action possibilities match between a person and an object [1]. Functional tones have, however, thus far remained obscure among practitioners, despite functional tones having a stronger theoretical foundation and facilitates a deeper and more humancentred analysis of interaction. The functional tones related to an object depend not only on the modes of sensation and action the perceiver is capable of, but also more subjective aspects such as experience, motivation and emotions. Using functional tones in design or analysis of interaction provides a fundamentally user experience centred perspective while avoiding the philosophical luggage of affordances.
\end{abstract}

Keywords. Functional tones, Affordances, Usability evaluation, User experience design, UXD.

\section{Introduction}

Whether designing a product to be manufactured, or designing tools for the production process itself it is important to identify how the intended and possible uses of the designed artefact are conveyed, i.e. how the user will understand the use of the artefact. The term affordance is often used for this; a door handle might be pull-able and a button might be press-able. The term affordances is borrowed from ecological psychology, and have since then taken on a life on its own and a plethora of interpretations have emerged $[1,2,3]$. We argue that functional tones, which is rooted in a theory called Umweltlehre, is often more appropriate and useful for human-centred design. Although affordances are said to exist in the relationship between a user and their environment, they are often discussed in terms of what the object can do for the user, what properties the object has [1]. Functional tones, on the other hand, are aspects in the subjective experiences of the user [4], and designing using functional tones will therefore force a fundamentally user experience centred perspective. Functional tones are fundamentally multi-modal, so 'tone' should be understood as nuance or flavour, in a modal agnostic sense. Understanding the larger theoretical context from which the terms are derived can thus

${ }^{1}$ Corresponding Author. erik.lagerstedt@his.se 
steer the design process by highlighting certain perspectives and facilitate certain options. We further argue that the use of appropriate concepts for understanding, designing, and evaluating how to use an object within a manufacturing facility has become even more critical now in the time of the high information load created by Industry 4.0, especially if the goal is to support the proposed Operator 4.0 framework [5].

This paper introduces functional tones as a concept that can be used to understand and evaluate how a human can understand interaction opportunities with products, objects, and their environment. This leads to a short but pragmatically framed discussion on how functional tones can affect design for interaction, as well as how this differs from the popular concept of affordances.

\subsection{Affordances}

To perceive an object's afforance is to perceive the function of or potential interactions with that object. This concept was introduced by Gibson in 1966 as a substitute for the philosophically contentious term value [6]; the affordance is in a sense the practical value the perceived object has for the perceiver. Gibson later elaborated on this further by stating that 'the affordance of anything is a specific combination of the properties of its substance and its surfaces taken with reference to an animal' [7, p. 67]. Affordances is a concept used in ecological psychology which emphasise pragmatism and situatedness to understand behaviour. The concept of affordances was picked up in the field of design by Norman [1], but was used in a slightly different way in that field; as inherent properties of an object that suggest how the object can be interacted with. Norman later clarified further that his use of the term referred to the subset of the affordances used by users as clues, and chose to rename those as signifiers [8]. These multiple interpretations of the term affordances has led to similar problems as the multiple meanings of value had for Gibson. Although affordances have been hotly debated in the scientific community, the fields of usability, design, and later user experience design (UXD) have made good use of the term while ignoring problems with the definition or the theoretical foundation $[9,10]$.

\section{Umweltlehre}

The concept of functional tones come from the theoretical work (known as Umweltlehre) of biologist Jakob von Uexküll $[11,4]$. Some theories and assumptions that Umweltlehre was strongly associated with in the early 20th century have since been refuted, however, many of the core concepts and principles have remained relevant. For instance has Umweltlehre had an important role in the development of ethology; the scientific study of animal behaviour primarily under natural conditions. Understanding behaviour in reallife situations based on the needs and desires of the individual provides perspectives and validity that is desirable also in design and manufacturing of artefacts for human use. In cognitive science many of the ideas important in Umweltlehre received some wider attention in the late 1980s [12], and is currently reemerging more explicitly. One interesting contribution that has been proposed is that Umweltlehre can be used to bridge the different, and sometimes conflicting, perspectives of ecological psychology [13] and enactivism [14], to form a basis for a general theory of cognition [15]. 


\subsection{Umwelt}

A key concept in Umweltlehre, after which it has taken its name, is the Umwelt, which could be directly translated as world around or surroundings/environment but is used to denote the subjective surroundings of an individual based on their sersorimotor capabilities as well as their affects, desires, etc. Today, the term Umwelt is often used in two similar but different ways [16]. In the first interpretation the Umwelt is the subset of the physical world that is accessible to the perceiver, whereas the second interpretation is that Umwelt is the perceiver's subjective experience of the world. The first interpretation can be seen as useful, albeit not particularly enlightening, and the second interpretation is richer and more in line with the original meaning and theoretical context.

In the case of the first interpretation, the Umwelt is the subset of the physical world; the things that are noticeable with the sensors and manipulable with the actuators of the individual in question. A wall can be part of a human's Umwelt as an obstacle, but the same wall can be part of a fly's Umwelt as a seat. The Umwelts are, however, not simply generalisable for the entire species; the wall that was an obstacle for one human can be a challenging exercise opportunity for another.

For the second interpretation, the constructivist aspects of Umweltlehre are more prominent. The world is experienced in a subjective way, continuously and unconsciously constructed by the perceiver based on their capabilities, their history of interactions and experiences, as well as current needs, desires, and goals. It might be the rest-ability of a bench rather than the challenge of a climbing wall that stands out to a tired climber. The Umwelt is thus continuously changing based on the current situation and its circumstances.

\subsection{Functional Tones, Affordances and the Umwelt}

Functional tones can be seen as Umweltlehre's counterpart to affordances. With the first, and more trivial, interpretation of Umwelt, the functional tones are the subset of the affordances remaining after they are filtered through the physical capabilities the perceiver. They are from this perspective similar to Norman's signifiers [8].

For the second interpretation, the functional tones are that which stands out to the perceiver, and an object can assume different tones depending on the affects and goals of the perceiver. A bike might, for instance, stand out as a mode of transportation or as a coat rack based on the needs of the perceiver. Again, these tones are individual since the history of interactions and experiences differs from person to person. As von Uexküll points out in an example, a guide can utilise aspects of the environment that is invisible to another person [4]. Part of the environment thus assumes a path tone to the guide.

Although the difference to affordances is clearer with the second kind of interpretation of Umwelt, the functional tones are beneficial in both cases. In particular due to the way they force the perspective to be situated and human centred in a fundamental way. Affordances are, in contrast, much more separated from the perceiver, making the user a complement to the object that is in focus. To what extent affordances are part of the perceiver, object or in the relation is still debated to a large extent, which is a major part of the reason for the heated and often destructive debates regarding the meaning of affordances. The strong realist position of Gibson in his ecological perspective has made it difficult to understand and include more subjective or constructivist aspects of interaction and perception. Gibson rejected the phenomenological world which many of the theories contemporary to him relied on, and 
only accepted the physical world. Umweltlehre, on the other hand, acknowledges the physical world, but emphasises that it is not accessible to any individual, that instead relies on the phenomenological world that they continuously build. That is, only part of the physical world is perceivable for the individual and it is therefore the experience of the physical world rather than the physical world itself that is meaningful for the perceiver.

An added benefit of taking the subjective aspects more seriously, is how aspect concerning user experience mesh smother and more natural.

\section{Functional Tones in Design}

We would like to highlight two topics where we see functional tones as particularly relevant for design and evaluation of interaction with technology. First to be discussed is the enactive perspective that functional tones inspire and how that forces a more human centred view during the design process or usability evaluation. Second, are some more specific examples of how functional tones can elucidate interactions with digital artefacts, in particular for augmented and virtual reality where affordances currently struggle.

\subsection{The Human Centred-ness}

When design uses functional tones it becomes necessary to analyse the Umwelt of the user as much as possible. This helps the design to keep an intrinsically human-centred viewpoint with particular attention to user experience at a deeper level, not just basic usability or efficiency. An artefact design under such conditions will be more likely to be framed in terms of how it solves the problem for the user, or more broadly how it contributes to the user experience. To design for certain functional tones is to have an empathetic perspective, which is central to UXD [10], and ensure that the artefact assumes the appropriate tones under the appropriate circumstances. It is the user that creates meaning through interaction, so the artefact should facilitate the user to perform their desired task. Moreover, the contextual nature of Umweltlehre and functional tones means that the artefact can take on different functional tones based on task, user, and context, which allows for analyses and evaluations that support more usage contexts as well as better supporting human diversity, both in a manufacturing context and in products to consumers.

Designing or analysing based on affordances will, in contrast, be primarily focused on the artefact. The artefact needs to be designed to have the right properties, and make sure to convey its properties and functions in a way that informs the user about possible ways to use it. An important contribution of affordances in design was to direct the blame regarding incorrect usage from the users to the designers, since the designers in those cases had failed to inform the users through the design what the correct usage is $[1,9]$. The problem still remains in the sense that the artefact has a correct way of being used, and it is up to the designer to make sure that the user get the right information to use it correctly. Functional tones, however, shift the focus instead onto the needs and circumstances of the user, which means that the designer can shift their attention from signalling how to use the artefact properly, to understanding the situated experience of the user and what is needed in that situation. 


\subsection{Functional Tones and Digital Artefacts}

A more specific situation where affordances have been particularly debated is when attempting to use the concept for virtual objects such as virtual buttons on a monitor or virtual objects in augmented or virtual reality [17]. An important aspect of the problem relates to whether an object's affordances can be rewritten by projections or similar illusions. The surface of a monitor has, for instance, the affordances of a vertical sheet of plastic, but a virtual button that is shown on screen has arguably a press-able affordance. This affordance is, however, only metaphorical, as the way to press this button is not to physically push on it (as long as it is not a touch-screen, which gives rise to new problems); a virtual cursor should be moved to its position, and a button at a completely different place should be pressed.

In the case of functional tones the perspective is more enactive, that is to say it is based on the view that humans make sense of the world around them based on a lifetime of experiencing it through interactions [18]. The meaning or reality of things around a human is therefore determined pragmatically rather than absolutely by emerging through interaction. An activity may consist of the user solving a problem, and pressing the virtual button is part of the process of solving that problem. With sufficient experience of interacting with computers the computer mouse becomes a part of the user's body, extending the reach of the user into the virtual world. The functional tones available from the virtual world can thus be explored through interaction, relying on continuous and parallel loops of action and perception to gain an understanding. This in turn means that objects that do not support the functional tones are expected to become less relevant or even lead to problems in interaction. What is real or not in a physical sense is of less importance, since the functional tones are aspects of the Umwelt; the subjective and phenomenological world of the specific user.

\subsection{Considering a Door}

To be a bit more concrete, let us consider a classic example in interaction design; a door. Doors and door handles are often used as typical examples when introducing and discussing affordances in design [1]. The shape of the handle can be shaped to afford griping and pulling or pushing, and designing with this in mind can provide information to the user about what direction the door swings without the user having to consider it explicitly. Having a handle that affords pulling on one side and a handle that affords pushing on the other will steer users to appropriate behaviour without explicit instructions. The start of the process of designing the door is thus with the door and the problem of conveying appropriate use. To solve that problem various features of the intended user are considered for exploitation, almost framing the user as something complementing the designed artefact.

With functional tones the design process instead starts with the user and their needs; in this case to enter and exit a building. However, with that information the analysis has barely started as entering or exiting isn't the purpose in itself. A person entering a hospital might want to visit a family member or might require medical attention. Such desires and circumstances can affect the state of mind and priorities of the user, which is necessary to take into account to design an appropriate door. This also means that it might be necessary to have different doors for different purposes since the functional tones will change with goals and affects of the users. An emergency door needs to be more, not less, noticeable for people in distress or panic, and after the door has revealed the escape 
path, it should make sure to also live up to being part of that path rather than something else, such as an obstacle. Resistance should in that case be kept to a minimum by, for instance, having the door swing out without any fiddly or time consuming mechanisms. After all, the user is not trying to open the door, they are trying to get out.

\section{Conclusions}

In this paper, we have briefly introduced functional tones, and its theoretical context Umweltlehre, as a concept to be used instead of or in parallel with affordances in design. Among the benefits of using functional tones is that it is fundamentally user experience centred. Instead of starting with the artefact and the problem of conveying the right way to use it, the starting point with functional tones is the subjective experience of the user, which frames the situation in which an artefact is potentially used. An added benefit of framing the artefact and its functions from the perspective of the user is that questions regarding things such as what is real and what is virtual, what is actually there and what is hidden, takes a secondary position, and more attention can be directed to the needs of the user.

\section{References}

[1] D.A. Norman. The psychology of everyday things. Basic books, New York, 1988.

[2] A. Chemero. An outline of a theory of affordances. Ecological psychology, 15 (2003), 181-195.

[3] F. Blin. The theory of affordances. Language-learner computer interactions: theory, methodology and CALL applications, (2016), 41-64.

[4] J. von Uexküll. A stroll through the worlds of animals and men: A picturebook of invisible worlds. In Claire H Schiller, editor, Instinctive Behavior - The development of a modern concept, International University Press, New York, (1957), 5-80.

[5] D. Romero, P. Bernus, O. Noran, J. Stahre, and Å. Fast-Berglund. The operator 4.0: human cyber-physical systems \& adaptive automation towards human-automation symbiosis work systems. In IFIP international conference on advances in production management systems, (2016), 677-686.

[6] J.J. Gibson. The Senses Considered as Perceptual Systems. Psychology Press, London, 1966.

[7] J.J. Gibson. The theory of affordances. In R.E. Shaw and J. Bransford, editors, Perceiving, acting, and knowing: Toward an ecological psychology, Lawrence Erlbaum Associates, (1977), 67-82.

[8] D.A. Norman. The way I see it: signifiers, not affordances. Interactions, 15 (2008), 18-19.

[9] A. Cooper, R. Reimann, D. Cronin, and C. Noessel. About face 4: the essentials of interaction design. John Wiley \& Sons, Indianapolis, 2014.

[10] R. Hartson and P.S. Pyla. The UX book: Agile UX design for a quality user experience. Morgan Kaufmann, Cambridge, 2018.

[11] J. von Uexküll. Teoretische Biologie. Springer, Berlin, 1928.

[12] H.R. Maturana and F.J. Varela. The tree of knowledge: The biological roots of human understanding. New Science Library/Shambhala Publications, 1987.

[13] J.J. Gibson. The ecological approach to visual perception: classic edition. Psychology Press, London, 2014.

[14] F.J. Varela, E. Thompson, and E. Rosch. The Embodied Mind, revised edition: Cognitive Science and Human Experience. MIT press, Cambridge, 2017.

[15] E. Baggs and A. Chemero. Radical embodiment in two directions. Synthese, (2018), 1-16.

[16] T.E. Feiten. Mind after Uexküll: a foray into the worlds of ecological psychologists and enactivists. Frontiers in psychology, 11 (2020), 1-10.

[17] J. Rambusch and T. Susi. The challenge of managing affordances in computer game play. Human IT: Journal for Information Technology Studies as a Human Science, 9 (2008), 83-109.

[18] D. Vernon. Enaction as a conceptual framework for developmental cognitive robotics. Journal of Behavioral Robotics, 1 (2010), 89-98. 